\title{
L'éducation musicale dans les Lois
}

Séline Gülgönen (USP)

This paper investigates musical education in Magnesia, as it is proposed in Platôิ Laws. Its role and nature are examined in its main parts. Musical education is supposed to begin before birth, in order to prepare the child as still a fetus to choral music. Such an effort has obviously a political aim, for choral music connects man to the cosmos, being thus the most appropriate way to cement the constitution, and provides a justification for the divine and human character of the constitution as well.

Je proposerai dans les pages qui suivent une interprétation de la nature et de la place de la chorée (choreia), art mêlant musique et danse, dans l'éducation des citoyens de Magnésie.

Destinée à l'ensemble des citoyens, hommes ou femmes, libres ou esclaves, du foetus $^{1}$ au vieillard ${ }^{2}$, la finalité de l'éducation des Lois est politique ${ }^{3}$ : elle doit modeler les âmes en orientant le plaisir qu'elles éprouvent ${ }^{4}$ dans une direction rationnelle, celle de la vertu ${ }^{5}$ édictée par la loi. La finalité de la musique se confond avec celle de l'éducation - la production de l'accord entre la raison et les affects selon la loi ${ }^{6}$-, de sorte que l'éducation musicale apparaît être un élément central de l'éducation générale, et, plus largement, pour la réalisation du projet de la cité. Je tenterai de démontrer

\footnotetext{
${ }^{1}$ VII, 792e.

2 II, 653c.

3 L'Athénien dit : «L'éducation, faut-il donc dire, consiste à attirer et à mener l'enfant dans la

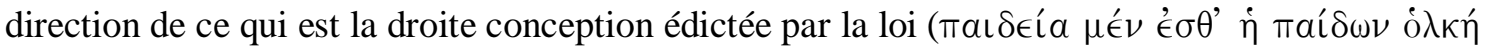

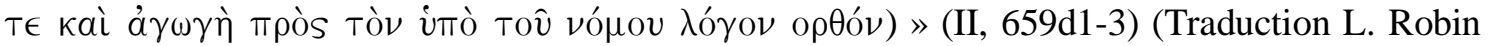
et J. Moreau, Paris, Gallimard, Bibliothèque de la Pléiade, 1950).

${ }^{4}$ II, 653b6-c4.

${ }^{5}$ II, $653 \mathrm{a}$.

${ }^{6}$ II, 659d1-e3.
} 
l'éducation musicale est essentiellement une éducation par la chorée, qui, de ce fait, est nécessaire au projet politique. Cette hypothèse a pour point de départ le lien de dépendance réciproque entre l'éducation et la participation à des choeurs ${ }^{7}$ énoncé au livre II :

«L'Athénien : (...) Allons-nous poser que l'éducation à ses débuts dépend des Muses et d'Apollon? Clinias : Oui, il en va ainsi. L'Athénien : Ainsi, pour nous, un homme dépourvu d'éducation sera celui qui ne fait pas partie d'un choeur, alors qu'il faut reconnaitre comme un homme éduqué celui qui a, comme il

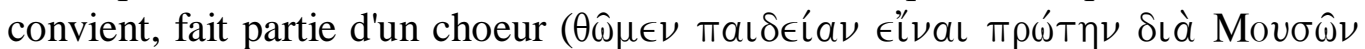

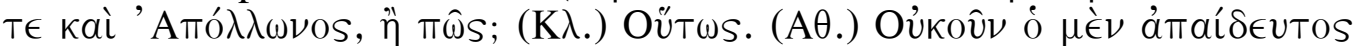

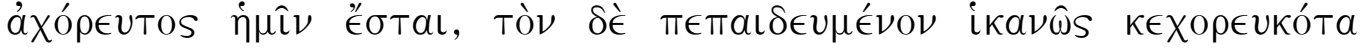
$\theta \in T \in ́ O \nu ;$ ) » (II, 654a6-b1).

Je m'attacherai à montrer que ce lien entre chorée et éducation doit être compris au sens propre, qu'il est établi au livre II parce que c'est à ce stade de l'argumentation que les interlocuteurs définissent l'essence même de l'éducation, et qu'il se trace implicitement tout au long du dialogue. La raison d'un tel lien se trouve dans la nature de la chorée, qui est, par essence, un art mimétique psychique et corporel.

G. R. Morrow ${ }^{8}$ puis M. B. Schoen-Lazzaro ${ }^{9}$ ont remarqué la nature imitative de la musique dans l'éducation, sans toutefois s'attarder cette qualité. La fonction des imitations musicale dans l'éducation a été en revanche étudiée récemment par E. JouëtPastré $^{10}$. Mon analyse au sujet du rôle et du fonctionnement de la musique dans l'éducation rejoint la sienne, mais je développerai certains aspects qu'elle n'envisage pas. Je me pencherai ainsi sur l'ensemble du cursus musical et chorégraphique proposé par l'Athénien, auquel à ma connaissance les interprètes n'ont pas prêté attention, et je chercherai à montrer que les disciplines dont il se compose n'existent que pour renforcer l'éducation par la chorée.

Il s'agit, ce faisant, de revenir sur l'idée communément admise et généralement

\footnotetext{
${ }^{7}$ Il s'agit de la chorée (choreia), qui « implique à la fois la danse et le chant» (II, 654b). Trad. L. Brisson et J. F. Pradeau (sauf indication contraire, nous utilisons cette traduction).

${ }^{8}$ G. Morrow, Platoô Cretan City ï A historical interpretation of the Laws (Princeton, Princeton University Press, 1960).

${ }^{9}$ M. B. Schoen-Nazzaro, «Plato and Aristotle on the ends of music » (Laval Théologique et Philosophique, Québec (Canada), Faculté de théologie de l'Université Laval, 1978).

${ }^{10}$ E. Jouët-Pastré, Le jeu et le sérieux dans les Lois de Platon (Sankt Augustin, Academia Verlag, 2006), p. 67-74.
} 
fondée sur une lecture exclusive du livre X de la République, selon laquelle Platon se ferait détracteur de l'ensemble des arts mimétiques, pour montrer que la chorée est à ses yeux l'art politique privilégié.

Nature et place de la chorée dans la législation de Magnésie

En confrontant plusieurs passages des livres II et VII, on peut s'apercevoir que la musique est avant tout conçue comme un art mimétique (technè mimetikè). Au livre II, l'Athénien explique que chacune des composantes de la chorée (rythmes, modes (harmoniai) et figures chorégraphiques) imite (mimeisthai) des «manières d'être» $(\text { tropoi })^{11}$, qui sont des caractères, des états psychologiques et corporels découlant du caractère du musicien ${ }^{12}$. L'Athénien ajoute que le législateur persuadera ou forcera les poètes à : «mettre dans les rythmes les figures [chorégraphiques], et dans les modes les mélodies d'hommes tempérants, courageux et pourvus de toutes les qualités morales (Tà

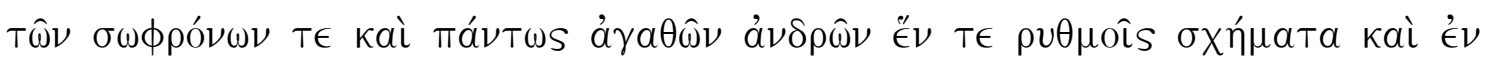

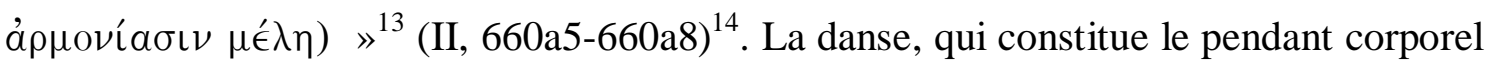
de la musique, est elle aussi mimétique, et ses modèles correspondent à ceux des mélodies et des rythmes musicaux. Au livre VII, l'Athénien évoque ainsi plusieurs danses, dont les descriptions s'avèrent se confondre avec celles de leurs modèles : la danse pyrrhique imite les mouvements des corps et des âmes des hommes courageux, tandis que la danse pacifique imite les mouvements des hommes dont l'âme est tempérante $^{15}$; d'autres danses, rejetées de Magnésie, imitent l'ébriété ${ }^{16}$.

\footnotetext{
${ }^{11} \mathrm{II}, 655 \mathrm{~d}$.

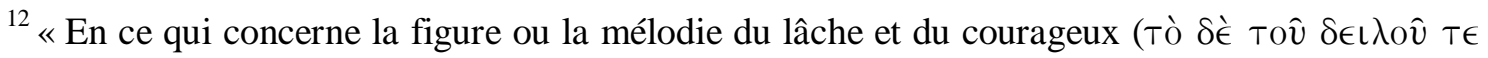

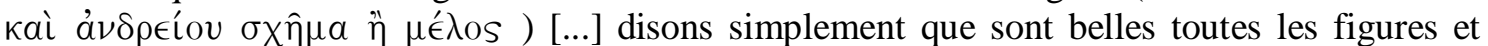
toutes les mélodies qui procèdent de l'excellence du corps ou de l'âme ( $\dot{\rho} \rho \epsilon \hat{\eta} s$ ÉXó $\mu \in \nu a$

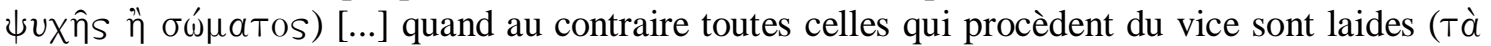

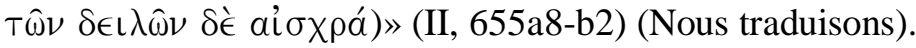

${ }^{13}$ Traduction L. Brisson et J.-Fr. Pradeau modifiée.

${ }^{14}$ Ceci est répété en VII, 798d8-e6.

${ }^{15}$ VII, 814e3-815a2.

${ }^{16}$ Ce sont: «la danse de caractère bachique, ainsi que celle auxquelles, sous les noms de «Nymphes », de «Pans », de «Silènes » et de «Satyres» se livrent des gens qui [...] imitent,
} 
L'importance des imitations musicales et chorégraphiques découle, en premier lieu, de l'importance de leurs modèles, dans le projet de la législation. Au livre I, les interlocuteurs établissent en effet que la finalité de la législation se confond avec la vertu, cette dernière formant un ensemble comprenant courage, justice, tempérance et réflexion. Or la musique et la danse éducatives imitent des vertus, qui sont le courage (andreia) et la tempérance (sophrosunè) ${ }^{17}$, ainsi que «toutes les qualités morales », que l'Athénien ne spécifie pas. La description des danses, en particulier, ne fait intervenir que courage et tempérance.

Lorsque les interlocuteurs examinent la nature du courage, ils concluent qu'une telle vertu se compose de deux parties complémentaires, l'andreia proprement dite, la résistance à la peur, mais aussi l'aidos, qui est le contrôle des plaisirs ${ }^{18}$; sans la seconde, l'andreia se révèle nuisible et perd sa qualité de vertu. Si les interlocuteurs désignent l'andreia comme modèle musical, au livre VIII, l'Etranger évoque aussi certaines

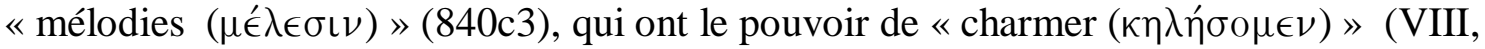

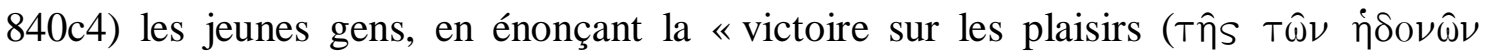

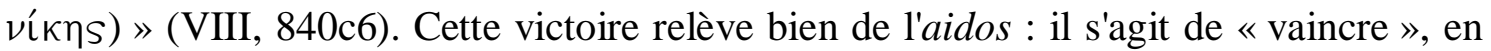
particulier, les plaisirs sexuels qui sont l'objet de l'analyse des interlocuteurs.

La tempérance ${ }^{19}$ est quant à elle d'emblée mise en rapport avec la musique. Elle reçoit alors une acception commune, la modération dans les plaisirs, en particulier sexuels ou relatifs à la boisson ${ }^{20}$, ainsi que la modération dans la richesse ${ }^{21}$. Au livre III,

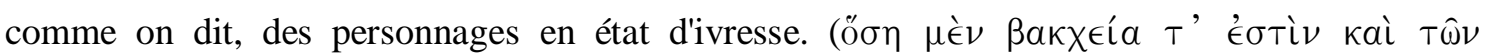

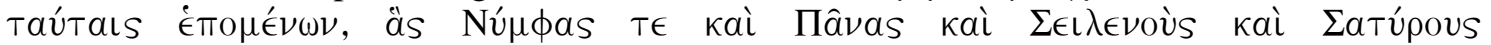

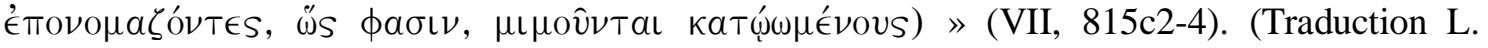
Robin modifiée).

${ }^{17}$ II, 655a8-9, 660a6-b1 pour la musique ; VII, 815d-e pour la danse.

${ }^{18} \mathrm{I}, 634-\mathrm{a}-\mathrm{b}$.

${ }^{19}$ Analysée à trois reprises : I, 635e-650 ; III, 696 a-e ; V, 729a-c.

${ }^{20}$ Voir aussi République, III. D. Carr montre qu'une telle définition de la tempérance l'apparente au courage, au sens où tous deux relèvent d'une maîtrise de la sensation physique. Selon lui, la différence tient alors au choix que permet la tempérance (entre différents plaisirs), alors que le courage n'implique pas de choix («The cardinal virtues and Plato's moral psychology », The Philosophical Quaterly, vol. 38, n 151, Saint Andrews, University of Saint Andrews, 1988, p. 192).

${ }^{21} \mathrm{~V}, 729 \mathrm{a}-\mathrm{c}$. 
les interlocuteurs en donnent toutefois une autre définition, spécifique aux Lois. Ils ne font alors plus référence à des pratiques existantes, mais établissent que la tempérance est une vertu «en surcroît $(\pi \rho o ́ \sigma \theta \eta \mu \alpha) »$ (III, 696d11), qui rend les caractères vertueux ${ }^{22}$. L'Athénien prend l'exemple du courage et de la justice ${ }^{23}$ : sans tempérance, aucun de ces caractères ne peuvent être vertueux. Plus précisément, la tempérance garantit que le plaisir accompagnant les affections humaines ne soit pas excessif, ce qui empêcherait le bon fonctionnement des autres vertus. Cette définition de la tempérance confirme le rôle essentiel de l'éducation musicale des citoyens de Magnésie, qui forme à la vertu la plus importante de l'éducation, en permettant le contrôle des plaisirs.

Etant donné le lien nécessaire entre courage, tempérance, sagesse et justice propre aux Lois $^{24}$, on est de plus amenée à penser que l'éducation au courage et à la tempérance implique aussi l'intelligence (phronèsis) et la justice (dikaiosunè). C'est de cette manière que l'on comprendra que l'Etranger évoque, pour la musique, «toutes les qualités morales ».

L'importance des vertus imitées par la chorée dans le projet législatif n'est toutefois pas suffisante pour justifier sa place centrale dans l'éducation. C'est en réalité la conjonction entre les qualités plaisante et imitative de la chorée - la première étant naturelle, l'autre relevant d'un «don » divin à partir du moment où elle imite un ordre qui paraît justifier la place centrale de cet art dans l'éducation ${ }^{25}$. Si elle était plaisante sans être imitative, la chorée ne pourrait en effet pas conduire à la vertu ; mais si elle

\footnotetext{
${ }^{22}$ III, 696 a-e.

${ }^{23}$ III, 696b-c.

${ }^{24}$ I, 631b-632a ; III, 688a-b ; IV, 709e-710a ; VIII, 836d ; XII, 962d ; 965b-d. Ce point a été mis en évidence par D. Carr, dans «The cardinal virtues and Plato's moral psychology» (The Philosophical Quaterly, op. cit.), ainsi que par L. Brisson et J. Fr. Pradeau, Les Lois, I, op. cit., p. 338 , n. 30 .

${ }^{25}$ Nous nous séparons sur ce point de Fr. Lasserre, qui montre que si l'imitation est un principe de l'éducation musicale dans la République, il n'en est pas de même dans les Lois, qui envisagent la musique en tant qu'elle est plaisante (Pseudo-Plutarque, De la musique, De la musique, Olten et Lausanne, Urs Graf-Verlag, 1954). Il nous semble au contraire que, s'il est vrai que le plaisir occupe dans les Lois une place qu'il n'avait en aucune façon dans la République, un tel plaisir est intimement lié à l'imitation que met en oeuvre la musique dont le caractère imitatif est explicite à plusieurs reprises.
} 
n'était qu'imitative, elle ne pourrait pas modeler les âmes. Le plaisir pris à la fréquentation des imitations de la chorée crée en effet des habitudes susceptibles de se transformer en nature : «il est assurément forcé que celui qui accueille [certaines mélodies et figures] avec plaisir devienne semblable à ces [...] figures ou mélodies qui

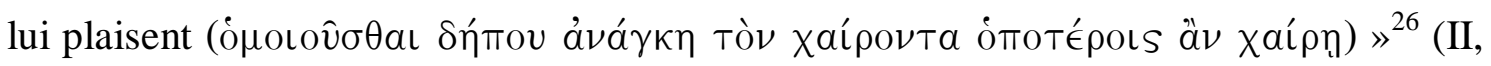
656b4-5). Devenir «semblable à des figures et à des mélodies» est une formule ramassée où « figures et mélodies » renvoient au caractère qu'elles imitent. Ce pouvoir de la musique est fondé anthropologiquement : les enfants, comme tous les jeunes êtres vivants, ont un sens inné du mouvement et du $\operatorname{son}^{27}$. Contrairement à ceux des autres animaux, leurs mouvements corporels peuvent toutefois être ordonnés, et ce, par le rythme, tandis que leur voix (définie comme leurs mouvements sonores) peut l'être par l'harmonie (harmonia), qu'il faut comprendre comme l'agencement des notes musicales au sein des modes. Or rythme (musical et chorégraphique) et harmonie (musicale) sont précisément les éléments de la chorée, qui, selon l'Athénien, constitue le prolongement divin $^{28}$ du sens inné du mouvement et du son ${ }^{29}$.

Pour que l'articulation entre qualité plaisante et imitative soit conforme au projet de la législation, le plaisir éprouvé doit être correct, autrement dit il ne doit être pris qu'à certaines imitations. Or, évaluer le bon plaisir musical apparaît d'autant plus problématique que le plaisir musical paraît, dans sa définition même, ne pouvoir être évalué : le plaisir est naturellement mouvant ${ }^{30}$, et la musique instrumentale se caractérise à son tour par des changements, comme l'atteste la description d'un apprentissage de la lyre qui dépasserait trois années ${ }^{31}$ sur lequel nous reviendrons. Dès lors, l'appréciation de la "bonne musique" est laissée aux hommes les plus vertueux ${ }^{32}$

\footnotetext{
${ }^{26}$ Traduction L. Brisson et J. Fr. Pradeau.

${ }^{27}$ II, 653d7-e3 ; 672b8-c5.

${ }^{28}$ II, 653e3-654a2.

${ }^{29}$ II, 654a2-5 ; II, 664e-665a ; 672b-d.

${ }^{30} \mathrm{II}, 657 \mathrm{~b} 4-5$.

${ }^{31}$ VII, 812d4-e6.

${ }^{32}$ II, $658 \mathrm{e} 7-8$.
} 
qui sont les plus éduqués ${ }^{33}$, à savoir les plus âgés des choristes. Leur formation musicale leur permet en effet de permet de discerner l'adéquation entre les modes, les rythmes et les caractères imités ${ }^{34}$ et d'apprécier les vertus. Cette formation est détaillée au livre VII.

\section{Le cursus éducatif}

Au livre VII, les protagonistes des Lois réfléchissent sur les possibilités de réalisation de l'éducation qu'ils proposent : ils examinent ses contenus et légifèrent avec précision sur les disciplines qui le composent. Ils sont en effet compétents pour cette tâche : âgés de plus de cinquante ans, originaires de Crète, de Sparte et d'Athènes, ils peuvent comparer les éducations, contemporaines ou passées, de chacune de leurs cités. L'éducation musicale et chorégraphique du livre VII étoffe celle qui a été d'abord établie au livre II et se réduisait à la chorée, identifiée comme l'essence de l'éducation. Je montrerai que toutes les disciplines de l'éducation musicale et chorégraphique du livre VII sont implicitement subordonnées à la première.

Le livre VII des Lois s'ouvre sur l'affirmation de l'importance d'un premier apprentissage, qui relève en réalité d'un «modelage » (il est appelé «trophè », terme dont le sens premier est «alimentation»), pour les foetus et les nouveaux-nés. L'Athénien prescrit pour ces destinataires une «gymnastique $(\gamma v \mu \nu \alpha \sigma \tau \iota \kappa ́ v)$ (VII, 789b2) précise : les mères doivent se promener régulièrement lorsqu'elles sont enceintes puis, une fois l'enfant né, le porter jusqu'à ce qu'il ait atteint l'âge de deux ans. Elles sont ensuite remplacées par des nourrices, qui feront de même en attendant que les enfants puissent marcher seuls ${ }^{35}$. Une telle gymnastique a pour but de procurer aux nouveauxnés un mouvement régulier et continu. Lorsque les enfants ne parviennent pas à

\footnotetext{
${ }^{33}$ « La Muse la plus belle est celle qui charme les meilleurs, c'est-à-dire ceux qui ont reçu une éducation suffisante, et surtout la Muse qui arrive à charmer ne fût-ce qu'un seul individu, celui

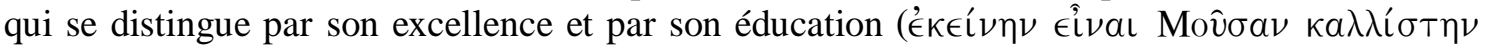

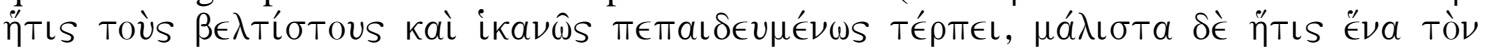

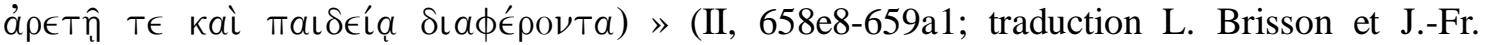
Pradeau).

${ }^{34}$ II, 670a7-b2.

${ }^{35}$ VII, 789e.
} 
s'endormir, le mouvement adapté pour les calmer est pendulaire, continu ${ }^{36}$ et régulier. Cette première «éducation » a pour finalité d'ordonner les gesticulations et les cris (par définition désordonnés rythmiquement et harmoniquement) des nouveaux-nés ${ }^{37}$. Ainsi, la «gymnastique » des nourrissons commence à infléchir leurs corps dans une direction rationnelle. On est dès lors amenée à faire l'hypothèse qu'une telle «gymnastique » constitue une propédeutique à la chorée ${ }^{38}$ : ce tout premier modelage (trophè) des corps en serait la première modalité.

D'autres indices permettent d'argumenter en faveur de cette lecture. Conformément à la conception de la gymnastique en Grèce ${ }^{39}$, la gymnastique est dans les Lois composée de la «danse» (orchèsis) et de la «lutte» (palè) $)^{40}$. Or la gymnastique des foetus et des nourrissons présente une parenté avec la danse : le balancement que procurent les mères et les nourrices aux enfants est mis en parallèle avec les mélodies apaisant les Corybantes possédés ${ }^{41}$, car tous deux présentent des

\footnotetext{
${ }^{36}$ VII, 790d.

${ }^{37}$ II, 653d.
}

${ }^{38}$ Ceci me conduit à me séparer de la position de M. S. Kochin, selon qui l'éducation des Lois commence alors que les enfants sont des corps malléables, et que sa première discipline est la gymnastique, tandis que celle de la République commence alors qu'ils sont des âmes malléables, par la musique («Unity of virtue and limitations of Magnesia », op. cit., p. 131). En réalité, il n'y a pas sur ce point une réelle opposition entre les deux éducations, qui toutes deux commencent par la musique. Cette dernière est composée d'une partie rythmique, plus corporelle bien qu'elle fasse aussi intervenir le logos (cette musique est celle de la première éducation des Lois) et d'une partie vocale, qui est moins corporelle, bien qu'elle le soit aussi parce qu'elle relève de la voix (cette musique est celle de la première éducation de la République).

${ }^{39}$ L. Séchan montre que de manière générale, la danse était en Grèce étroitement liée à la gymnastique : «la danse grecque est plus uniformément active que la nôtre pour l'ensemble de l'organisme ; elle est beaucoup moins subordonnée aux seuls mouvements des jambes [...] Elle avait, à ce titre, le rapport le plus étroit avec la gymnastique. De nombreux mouvements, à la fois gymnique et orchestique, étaient exécutés au son de la flûte (aulos) par les enfants à la palestre [...] C'était à la palestre et dans les gymnases qu'on apprenait, notamment, les principes de la pyrrhique et de la chironomie qui, au sens gymnique du mot, consistait dans l'exécution, sur un rythme, des mouvements de la danse et du combat » (La danse grecque antique, éd. De Broccard, Paris, 1930, p. 64-65). L'auteur montre que la différence entre gymnastique et danse tient à l'expressivité de la seconde, exécutée en vue de "produire un effet» (p. 66). En particulier, les schèmata relèvent de la danse et non plus de la gymnastique.

${ }^{40}$ II, 673a-d ; VII, 795d.

${ }^{41}$ VII, 790e. 
mouvements ordonnés et réguliers. La définition du rythme qui en fait l'« ordre du

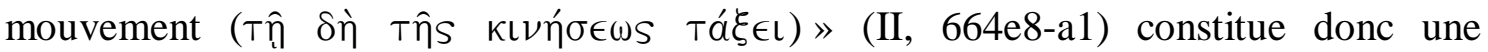
description exacte de la trophè. L'« ordre » impliqué par le rythme constitue la raison pour laquelle les mouvements procurés aux foetus et aux nouveaux-nés sont éducatifs.

$\mathrm{Au}$ livre $\mathrm{X}$, dans son analyse des mouvements, l'Athénien rapproche le mouvement pendulaire du mouvement circulaire, identifié comme celui de la pensée ainsi que comme celui des astres ${ }^{42}$, qui sont divins. Tout en lui étant inférieur, le mouvement pendulaire a en commun avec le mouvement par rotation le fait que chacun des points mus revient au même emplacement. La régularité des mouvements de la trophè peut aussi être mise en rapport avec la régularité des mouvements circulaires de la pensée et des astres : elle tend à accorder les corps avec le mouvement du cosmos et de la rationalité.

La trophè constitue donc une première étape de la partie corporelle de la chorée (la danse), en constituant une sorte d'initiation passive à la danse pour les foetus et les nouveaux-nés. Elle ne fait que commencer à former leurs corps, en leur procurant un terrain sur lequel pourront se développer les imitations plus précises de la chorée. Cette première phase de l'éducation est sans doute la plus profonde, parce qu'elle présente une dimension cosmique et universelle. Elle est ainsi nécessaire à l'éducation musicale qui suivra, dont la forme déterminée est celle que veut la législation de Magnésie.

Après avoir reçu une première éducation rythmique, une fois qu'ils ont appris à parler, les enfants doivent pratiquer l'art choral et poursuivre cet apprentissage tout au long de leur vie. L'Athénien institue pour cela trois choeurs par classes d'âge, celui des enfants, celui des jeunes gens, et celui des «vieillards ». La caractérisation de chacun des choeurs développe la description de la fonction de la chorée (livre II) : les choeurs doivent former à l'excellence, c'est-à-dire accorder le plaisir et la raison. La musique et la danse des choeurs sont imitatives, et leurs imitations des vertus doivent s'accorder avec le sens des paroles ${ }^{43}$.

Pour chaque classe d'âge, la chorée joue bien un rôle pédagogique. L'art choral

\footnotetext{
${ }^{42} \mathrm{X}, 898 \mathrm{a} 8-\mathrm{b} 3$.

${ }^{43}$ II, 664c.
} 
est avant tout destiné à modeler les âmes des plus jeunes : «L'ensemble des choeurs, qui sont au nombre de trois, doivent adresser leurs incantations aux âmes des enfants, tant

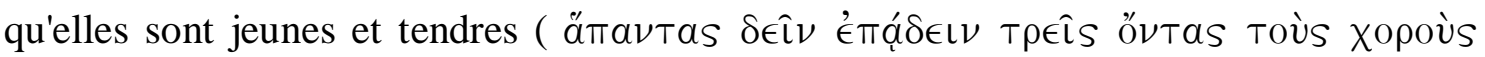

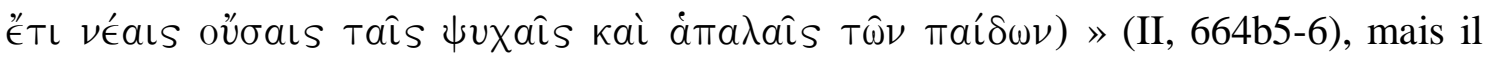
doit aussi éduquer les membres des autres choeurs. De ce point de vue, les hommes du troisième choeur, qui ont entre trente et soixante ans, reçoivent un traitement particulier : ils doivent chanter en état d'ébriété ï voilà pourquoi le dieu tutélaire de ce choeur est Dionysos ${ }^{44}$. En effet, l'Athénien explique que la honte empêcherait les personnes âgées de chanter, si elles n'étaient pas ivres ${ }^{45}$. L'ivresse du troisième choeur est en effet la condition de leur malléabilité :

« les âmes de ceux qui boivent deviennent, lorsqu'elles sont soumises à ce feu, comme c'est le cas pour un morceau de fer, plus souples et plus jeunes, au point de se laisser manier par celui qui peut et sait les éduquer et les façonner, comme

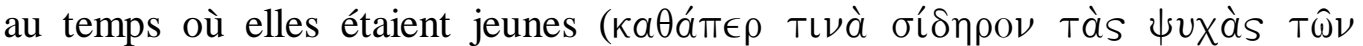

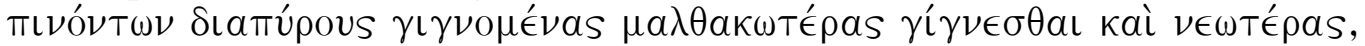

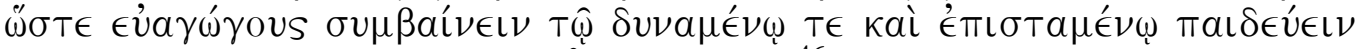

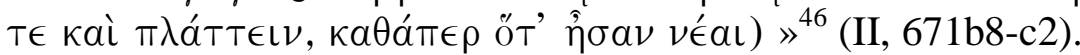

Un tel jugement positif sur l'ivresse ${ }^{47}$, généralement condamnée, s'explique par la caractérisation des choristes, dont il faut remédier aux faiblesses de caractère récurrentes, et qui doivent pour cela être éduqués tout au long de la vie de la même manière. L'ivresse est ce qui conduit les vieillards à retrouver un état d'enfance, c'est-àdire un état qui leur permet d'être constamment éduqués.

Les interlocuteurs réservent ensuite une partie du programme éducatif à l'apprentissage de la lyre ${ }^{48}$, de treize à seize ans. Cet apprentissage a deux objectifs explicites : l'acquisition des connaissances nécessaires à la reconnaissance des modes et des rythmes imitatifs de la musique vocale ${ }^{49}$, et le placement de la voix en fonction de la

\footnotetext{
${ }^{44}$ II, 664d-665b.

${ }^{45}$ II, $672 \mathrm{~d}$.

${ }^{46}$ Traduction L. Brisson et J. Fr. Pradeau modifiée.

${ }^{47}$ Les interlocuteurs feront une réglementation de l'usage de la boisson au livre II. L'ivresse peut être utilisée avec profit dans le cas de la chorée.

${ }^{48}$ VII, 812d-813a.

${ }^{49}$ VII, 812c.
} 
tension des $\operatorname{cordes}^{50}$, autrement dit la justesse. La pratique de la lyre a donc pour fonction de développer d'une part l'écoute correcte des imitations musicales, et d'autre part leur production. Elle consolide la formation chorale.

Un tel enseignement a pour corollaire deux interdictions, l'une explicite et l'autre implicite, que l'on interprétera comme ayant pour fonction d'empêcher que ne se développent l'écoute et la production d'une musique non-imitative. La première interdiction, explicite, tient aux modalités strictement réglementées de l'apprentissage de la lyre : il ne peut dépasser trois années, et ne doit pas présenter de difficultés. L'apprentissage de la lyre, ainsi conçu, empêche le développement de la virtuosité instrumentale. Or on peut établir que la virtuosité, pour Platon, se confond avec le caractère non-imitatif de la musique. Voici comment l'Athénien décrit une musique virtuose :

«Par la suite cependant, avec le temps qui marchait, parurent des poètes qui furent les initiateurs des infractions aux lois de la musique; hommes naturellement doués sans doute pour la poésie, mais ignorants de ce que la Muse comporte de légitime et d'accrédité, possédé plus qu'il n'eût fallu, dans leurs transports, par la passion du plaisir et qui mélangèrent les "thrènes" aux "hymnes", les "péans" aux "dithyrambes" ; imitant sur la cithare l'accompagnement du chant par l'aulos ; amenant tout à se confondre avec tout ; prétendant mensongèrement, dans leur involontaire déraison, qu'en musique il n'y a même pas place pour une rectitude quelconque et que, hormis le plaisir de celui qui y trouve sa jouissance, il n'existe pas de moyen plus correct de

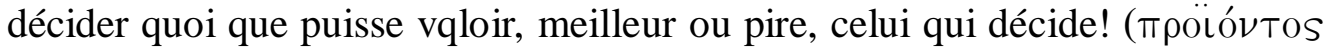

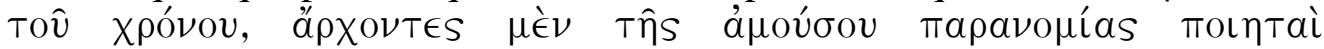

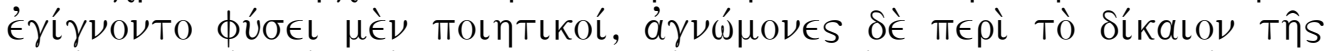

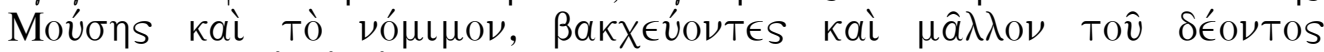

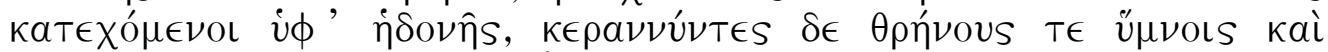

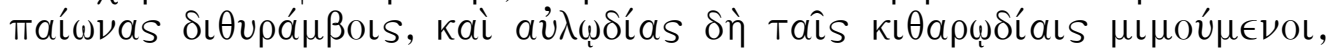

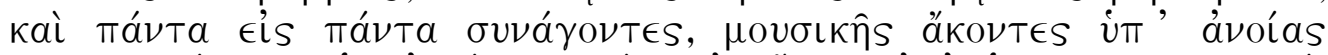

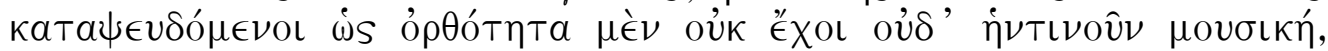

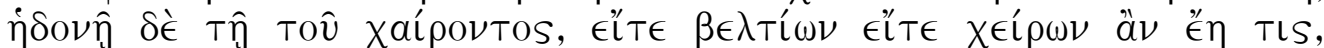

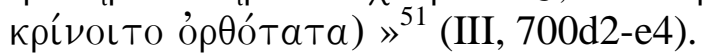

Une mauvaise musique provoque un plaisir excessif. Or l'excès de plaisir musical renvoie à un défaut de rectitude, se confondant avec un défaut d'imitation. La mauvaise musique multiplie en effet les éléments musicaux, le mélange des instruments

\footnotetext{
${ }^{50}$ VII, 812d3-4.

${ }^{51}$ Traduction L. Robin. Nous modifions « flûte » par « aulos ».
} 
impliquant celui des modes et des rythmes, ainsi que celui des genres (chaque instrument joue de manière privilégiée certains modes et certains genres $)^{52}$. De tels mélanges ne peuvent produire des imitations, ni le plaisir qui en découle être vertueux. Un apprentissage poussé de la lyre conduirait à jouer de cette musique non-imitative.

Mais un autre type d'apprentissage instrumental pourrait avoir les mêmes conséquences : il s'agit de celui de l'aulos, qui était habituel en Grèce ${ }^{53}$, mais n'apparaît pas dans le cursus éducatif des Lois. L'aulos se caractérise pour Platon avant tout par son approximation: l'Athénien remarque par exemple qu'il est impossible à une foule éduquée à danser au son de l'aulos de reconnaître les modèles de tel ou tel mode ${ }^{54}$. Autrement dit, son apprentissage n'éduquerait pas à l'identification des imitations musicales. L'aulos est de surcroît incompatible avec le travail sur la justesse de la voix qui est un des objectifs de la formation instrumentale des Lois, et requiert la stabilité des instruments à cordes : l'apprentissage de l'aulétique ne mènerait donc pas non plus à la production des imitations musicales.

L'éducation instrumentale des Lois, limitée à l'apprentissage de la lyre, paraît donc avoir pour seule finalité la consolidation de la partie sonore de la musique chorale et imitative. Elle est subordonnée aux paroles des chants des choeurs, et conçue de manière à empêcher l'apprentissage ou l'écoute de la musique non-imitative.

La partie corporelle de la chorée, la danse, est à son tour consolidée par une formation spécifique. Au livre VII, les protagonistes établissent que la paideia doit comporter une formation des corps par la gymnastique et une formation des âmes par l'art des Muses (qui comprend la musique, mais aussi la poésie) ${ }^{55}$. Les enfants doivent recevoir, comme formation physique, une formation à la gymnastique, qui se compose de lutte (palè) et de danse (orchèsis) ${ }^{56}$. Cette dernière est précisément une éducation rythmique. Suite à la trophè des foetus et des nouveaux-nés, cet apprentissage constitue

\footnotetext{
${ }^{52}$ République, III.

${ }^{53}$ Fr. Lasserre, Pseudo Plutarque, De la Musique, op. cit.

${ }^{54}$ II, 670b-c.

${ }^{55}$ VII, 795c.

56 VII, 795d. Au livre II, l'analyse de la danse coïncide avec une analyse rapide de la gymnastique (II, 673a-d).
} 
ainsi la première phase de l'éducation chorégraphique.

Après cette éducation physique, les interlocuteurs posent la nécessité d'une formation proprement chorégraphique. L'Athénien rappelle l'importance de la «danse armée » dans l'éducation de la chorée :

«Pas davantage ne devons-nous négliger tout ce qui encore dans les choeurs constitue une imitqtion appropriée à notre but ; ainsi, dans le pays où nous sommes, les divertissements armés des Courètes et, à Lacédémones, ceux des

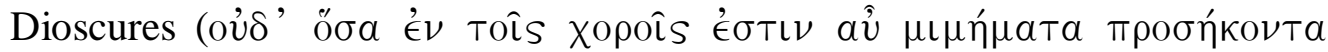

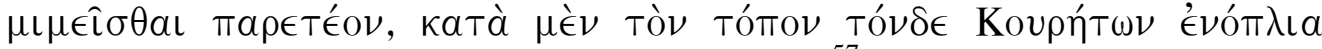

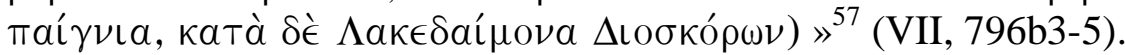

Les danses armées forment les jeunes gens au courage. Elles possèdent certaines qualités propres à la danse et d'autres spécifiques à la lutte. Leur caractérisation est rythmique, et en ce sens elles sont précisément chorégraphiques : les danseurs, lors de leurs prestations, doivent «donn[er] aux supplications que, par leurs danses et par leurs marches, ils adressent aux dieux et aux enfants des dieux, une cadence plus rapide ou

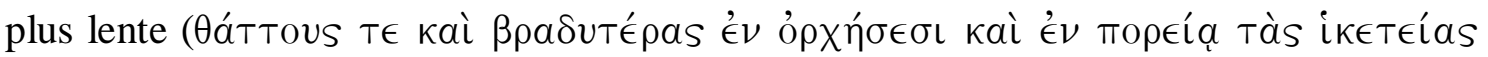

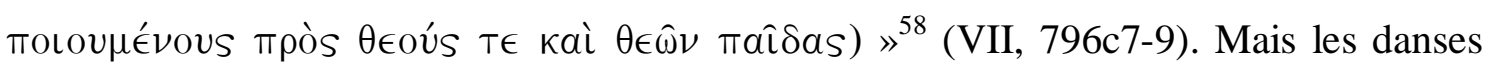
armées relèvent aussi de la lutte : leur modèle est Athéna, la déesse de la guerre :

«Chez nous aussi, d'autre part, la Vierge-Reine [Athéna], ayant trouvé du charme au divertissement de la danse, ne pensa pas s'amuser à ce jeu les mains vides ; mais, s'étant parée de son armure complète, c'est ainsi qu'elle voulut se

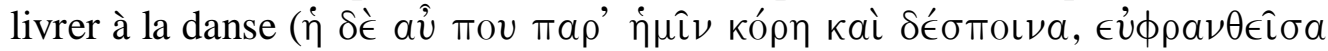

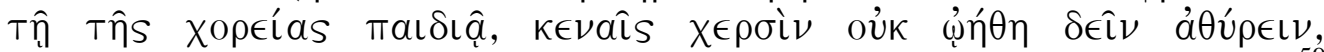

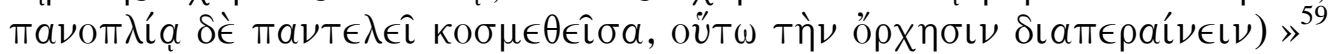
(VII, 796b6-c2).

Jeunes filles et jeunes hommes doivent «imiter ( $\mu \iota \mu \epsilon \hat{\imath} \sigma \theta a \iota) »(V I I, 796 c 2)$ cette déesse exemplaire, au sens où il s'agit de la prendre pour modèle et d'agir de la façon dont elle agit, par le biais de l'imitation chorégraphique qui reçoit la fonction précise d'une technique de transformation: les danses armées rendent ceux qui les exécutent courageux, et leur spectacularisation, par le biais des processions et des cortèges, contribue à développer le courage des spectateurs. La danse armée devient, pour toutes

\footnotetext{
${ }^{57}$ Traduction L. Robin.

${ }^{58}$ Traduction L. Robin.

${ }^{59}$ Traduction L. Robin.
} 
et tous, un moyen de célébrer à la fois la religion et la guerre ${ }^{60}$.

Les interlocuteurs reviennent ensuite sur la danse, qu'ils considèrent alors comme une partie de l'éducation par l'art des Muses. L'Athénien et Clinias décrivent les danses guerrières et les danses pacifiques, imitant le courage et la tempérance, intégrées au cursus éducatif. Cette éducation chorégraphique doit être comprise comme une éducation rythmique générale. E. Benvéniste a montré dans un article célèbre que le sens du terme « rythme » prenait chez Platon un sens nouveau :

Platon emploie encore rhuthmos au sens de 'forme distinctive, disposition, proportion'. Il innove en l'appliquant à la forme du mouvement que le corps humain accomplit dans la danse, et à la disposition des figures en lesquelles ce mouvement se résout [...] Voilà le sens nouveau de ruthmos : la «disposition » (sens propre du mot) est chez Platon constituée par une séquence ordonnée de mouvements lents et rapides, de même que l'« harmonie résulte de l'alternance de l'aigu et du grave. ${ }^{61}$

L'éducation chorégraphique permet de comprendre une telle inflexion que Platon fait subir au terme : le philosophe applique le substantif de «rythme» à ce qui est en mouvement, qui relève d'un plaisir inné propre à tout être humain, et l'oriente vers un ordre, précisément celui que prend la forme de ses imitations. À la lumière de cette analyse, on peut revenir sur l'ensemble de l'éducation des corps. Le lien entre la trophè $\mathrm{du}$ foetus et du nourrisson et l'éducation chorégraphique paraît validé : la gymnastique imposée au foetus constitue une propédeutique à la danse et à l'éducation « rythmique », et il s'agit donc bien du «rythme» dans sa nouvelle acception platonicienne. Cette première éducation restait toutefois rudimentaire, puisque les mouvements n'y sont pas disposés selon le lent et le rapide et n'imitent pas des caractères précis, mais sont simplement réguliers $і ̈$ ils constituent l'ordre sur lequel peuvent se développer d'autres configurations rythmiques. Ce serait l'équivalent d'un apprentissage d'une pulsation régulière, nécessaire pour pouvoir par la suite apprendre à diviser le temps selon des formules rythmiques plus complexes, qui sont justement celles de la danse.

La danse éduque donc les corps, mais elle accompagne la musique vocale de la chorée, qui éduque les âmes. Le développement consacré à la danse armée confirme que

\footnotetext{
${ }^{60}$ VII, 796c.

${ }^{61}$ E. Benveniste, «La notion de ñrythmeò dans son expression linguistique » (Problèmes de linguistique générale, 1, Gallimard, 1966, p. 334 [Journal de psychologie, 1951]).
} 
les parties de l'éducation convergent vers la chorée, et que l'imitation musicale et chorégraphique, parce qu'elle est plaisante, constitue la méthode, mais aussi l'essence de l'éducation. Celle-ci se fait par le biais de l'imitation et se confond avec elle.

Le programme éducatif vocal, instrumental et chorégraphique des citoyens de Magnésie a pour objectif la formation des corps et des âmes. Il a pour noyau la chorée : l'éducation des foetus et des nourrissons en constitue une propédeutique, l'apprentissage instrumental consolide sa partie vocale, tandis que l'apprentissage de la danse consolide sa partie chorégraphique. Ces enseignements sont pensés en fonction des imitations des vertus spécifiques à la chorée. Ainsi, l'éducation des foetus et des nouveaux-nés imite un mouvement cosmique et rationnel, et prépare leur corps à pouvoir recevoir les imitations de la chorée, qui sont faites d'autres mouvements rationnels. L'éducation instrumentale est réglementée afin de ne servir que la musique imitative ; en effet, elle pourrait tendre vers la musique non-imitative plus que les autres parties de la chorée et, étant donné son efficacité, de manière plus dangereuse. Tout ce qui en elle pourrait conduire à une musique non-imitative (l'apprentissage de l'aulos et la virtuosité) est écarté. L'éducation chorégraphique permet enfin d'approfondir la partie corporelle des imitations de la chorée, en éduquant aux danses imitant le courage et la tempérance. La chorée, qui constitue le noyau de l'éducation des citoyens de Magnésie, est en ce sens essentielle à la Cité : elle est politique, et, parce qu'elle relie la nature de l'homme au cosmos, elle apparaît à la fois être la technique la plus appropriée pour asseoir la Constitution, et une des techniques justifiant son bien-fondé anthropologique et divin.

La critique adressée aux arts imitatifs, au livre $\mathrm{X}$ de la République ${ }^{62}$, que la plupart des commentateurs considèrent comme une critique radicale de l'imitation, témoignerait ainsi d'une évolution dans la pensée de Platon, ou bien doit être reconsidérée. Etant donné la complémentarité entre les deux dialogues, mise en évidence par plusieurs auteurs et en particulier A. Laks ${ }^{63}$, je propose de considérer le second terme de l'alternative. A relire le livre X, on s'aperçoit que les critiques adressées

\footnotetext{
${ }^{62}$ République, X, 595a5-b5.

${ }^{63}$ Par exemple «Legislation and Demiurgy : On the Relationship Between Plato's Republic and Laws », Classical Antiquity, Berkeley, University of California Press, 1990.
} 
aux arts mimétiques au livre $\mathrm{X}$ de la République concernent une imitation qui se voudrait concurrente de la philosophie ${ }^{64}$; par ailleurs, il n'est pas sûr que musique et danse, au sens restreint, soient visées par ces critiques (du moins, les interlocuteurs n'en parlent-ils jamais explicitement). Il semble que la chorée ne puisse qu'échapper aux critiques que Platon émet par l'intermédiaire de Socrate, dans ce dialogue. Loin de se poser comme concurrente de la philosophie, la chorée des Lois en est l'adjuvant, si la Constitution des Lois découle d'une connaissance philosophique de la nature de l'homme et de la société.

\section{Bibliographie}

Babut, D.«Sur la notion d'imitation dans les doctrines esthétiques de la Grèce classique », Revue des Etudes Grecques, 48, Paris, 1985.

Benveniste, E. «La notion de ñrythmeò dans son expression linguistique », Problèmes de linguistique générale, 1, Gallimard, 1966 [Journal de psychologie, 1951].

Brisson, L. \& Pradeau, J.-F. Platon : Les Lois. GF Flammarion, 2006.

Carr, D. «The cardinal virtues and Plato's moral psychology », The Philosophical Quaterly, vol. $38, \mathrm{n}^{\circ} 151,1988$.

Jouët-Pastré, E. Le jeu et le sérieux dans les Lois de Platon, Sankt Augustin, Academia Verlag, 2006.

Laks, A. «Legislation and Demiurgy : On the Relationship Between Plato's Republic and Laws », Classical Antiquity, Berkeley, University of California Press, 1990.

Lasserre, Fr. Pseudo-Plutarque, De la musique, Olten et Lausanne, Urs Graf-Verlag, 1954.

Morrow, G. Platô̂ Cretan City Ï A historical interpretation of the Laws, Princeton, Princeton University Press, 1960.

Schoen-Nazzaro, M. B. «Plato and Aristotle on the ends of music », Laval Théologique et Philosophique, Québec (Canada), Faculté de théologie de l'Université Laval, 1978.

Séchan, L. La danse grecque antique, éd. De Broccard, Paris, 1930.

${ }^{64}$ C'est notamment l'interprétation de D. Babut, «Sur la notion d'imitation dans les doctrines esthétiques de la Grèce classique »(Revue des Etudes Grecques, 48, Paris, 1985), p. 49. 